\title{
On the Wigner transform of solutions to the Schrödinger equation
}

\section{Diogo A. Gomes}

Departamento de Matemática, Instituto Superior Técnico, Av. Rovisco Pais, 1049-001 Lisboa, Portugal

\section{Jorge D. Silva}

Departamento de Matemática, Instituto Superior Técnico, Av. Rovisco Pais, 1049-001 Lisboa, Portugal

\begin{abstract}
In this paper we use the Wigner transform to study properties of solutions to the Schrödinger equation. In particular, we present an elementary proof of the time decay inequalities for the free particle and harmonic oscillator. Furthermore, we estimate certain localized integral quantities in phase space, involving the Wigner transform of solutions to the Schrödinger equation.
\end{abstract}

\section{Introduction}

The Wigner transform has been used by several authors to study the Schrödinger equation: as a fundamental tool to study the semiclassical limit [4, 5] and, by using multiplier techniques [3], to prove dispersivetype estimates. See also [1]. In this paper we establish certain phase space localization-type estimates for the Wigner transform of solutions to the Schrödinger equation.

This paper has the following structure. Firstly, we review the basic properties of the Wigner transform and obtain the transport equation that results from its application to the Schrödinger equation. Then, we discuss some explicit examples for which the Wigner transform yields elementary proofs of time-decay estimates for the fundamental solution of the corresponding Schrödinger equation: the free particle and the harmonic oscillator. In section 4 we develop an abstract approach to the study of the

Partially supported by the Center for Mathematical Analysis, Geometry and Dynamical Systems through FCT Program POCTI/FEDER and also by grant POCI/FEDER/MAT/55745/2004. 
Wigner equation and prove estimates for the evolution of certain integral quantities that involve the Wigner transform of solutions of the Schrödinger equation, localized in phase space by cutoff functions transported by the corresponding Hamiltonian flow.

\section{A review of the Wigner transform}

In this section we provide a fast overview of the basic properties of the Wigner transform and its connection to the Schrödinger equation.

2.1. Definitions and basic properties. A thorough and careful presentation of all these facts can be found in [2. The Wigner transform $W[f, g]$ of two functions $f, g \in \mathcal{S}\left(\mathbb{R}^{n}\right)$ is defined by the integral formula

$$
W[f, g](x, \xi)=\int_{\mathbb{R}^{n}} e^{-i \xi \cdot y} f\left(x+\frac{y}{2}\right) \bar{g}\left(x-\frac{y}{2}\right) d y,
$$

for $(x, \xi) \in \mathbb{R}^{n} \times \mathbb{R}^{n}$. This transform is clearly a continuous, sesquilinear map $W: \mathcal{S}\left(\mathbb{R}^{n}\right) \times \mathcal{S}\left(\mathbb{R}^{n}\right) \rightarrow \mathcal{S}\left(\mathbb{R}^{2 n}\right)$. By considering it as a linear operator acting on tensor products $f \otimes \bar{g}$ - simply a measure preserving linear change of variables followed by the Fourier transform in one of the variables only this definition is naturally extended to a map $\mathcal{S}^{\prime}\left(\mathbb{R}^{n}\right) \times \mathcal{S}^{\prime}\left(\mathbb{R}^{n}\right) \rightarrow \mathcal{S}^{\prime}\left(\mathbb{R}^{2 n}\right)$, whose restriction to $L^{2}\left(\mathbb{R}^{n}\right) \times L^{2}\left(\mathbb{R}^{n}\right)$ maps into $L^{2}\left(\mathbb{R}^{2 n}\right) \cap C_{0}\left(\mathbb{R}^{2 n}\right)$. From Parseval's identity for the Fourier transform one immediately obtains the so called Moyal identity

$$
\left\langle W\left[f_{1}, g_{1}\right], W\left[f_{2}, g_{2}\right]\right\rangle=(2 \pi)^{n}\left\langle f_{1}, f_{2}\right\rangle \overline{\left\langle g_{1}, g_{2}\right\rangle},
$$

which implies

$$
\|W[f, g]\|_{L^{2}}=(2 \pi)^{n / 2}\|f\|_{L^{2}}\|g\|_{L^{2}},
$$

whereas by applying Hölder's inequality to (1) one gets

$$
\|W[f, g]\|_{L^{\infty}\left(\mathbb{R}^{2 n}\right)} \leq 2^{n}\|f\|_{L^{2}\left(\mathbb{R}^{n}\right)}\|g\|_{L^{2}\left(\mathbb{R}^{n}\right)} .
$$

The Wigner transform applied to the Fourier transforms of $f$ and $g$ satisfies the property

$$
W[\hat{f}, \hat{g}](x, \xi)=(2 \pi)^{n} W[f, g](-\xi, x),
$$

where our definitions of the Fourier transform, and its inverse, are

$$
\hat{f}(\xi)=\int e^{-i \xi \cdot x} f(x) d x, \quad f(x)=\frac{1}{(2 \pi)^{n}} \int e^{i \xi \cdot x} \hat{f}(\xi) d \xi .
$$

An alternative representation for $W$, in terms of $\hat{f}$ and $\hat{g}$, is therefore given by

$$
W[f, g](x, \xi)=\frac{1}{(2 \pi)^{n}} \int e^{i p \cdot x} \hat{f}\left(\xi+\frac{p}{2}\right) \overline{\hat{g}}\left(\xi-\frac{p}{2}\right) d p .
$$


It is also clear that $W[f, g]=\overline{W[g, f]}$, so if $f=g$ the Wigner transform $W[f, f]$ is a real valued function which we denote simply by $W[f]$.

An important property is the following: integrating out either the position or momentum yields the marginal probability distribution of the other variable

$$
|f(x)|^{2}=\frac{1}{(2 \pi)^{n}} \int_{\mathbb{R}^{n}} W[f](x, \xi) d \xi, \text { and }|\hat{f}(\xi)|^{2}=\int_{\mathbb{R}^{n}} W[f](x, \xi) d x .
$$

From any of these two marginal distributions, one obtains

$$
\|f\|_{L^{2}\left(\mathbb{R}^{n}\right)}^{2}=\frac{1}{(2 \pi)^{n}} \iint_{\mathbb{R}^{2 n}} W[f](x, \xi) d \xi d x,
$$

while from Moyal's identity (2)

$$
\|f\|_{L^{2}\left(\mathbb{R}^{n}\right)}^{2}=\frac{1}{(2 \pi)^{n / 2}}\|W[f]\|_{L^{2}\left(\mathbb{R}^{2 n}\right)} .
$$

2.2. Schrödinger equation. Let $\psi$ be a solution to the Schrödinger equation

$$
i \partial_{t} \psi=-\frac{1}{2} \Delta \psi+V \psi
$$

with $V: \mathbb{R}^{n} \rightarrow \mathbb{R}$ a real valued potential. Throughout the paper we assume that $V$ has the form

$$
V(x)=P_{2}(x)+b(x), \quad x \in \mathbb{R}^{n},
$$

where $P_{2}$ is a polynomial of degree $\leq 2$ and $b \in \mathcal{S}\left(\mathbb{R}^{n}\right)$ is a perturbation.

Define $\kappa$ by the formula

$$
\begin{aligned}
\kappa(x, \xi) & =\frac{i}{(2 \pi)^{n}} \int_{\mathbb{R}^{n}}\left[V\left(x+\frac{y}{2}\right)-V\left(x-\frac{y}{2}\right)\right] e^{-i \xi \cdot y} d y \\
& =\frac{i}{\pi^{n}}\left[\hat{V}(2 \xi) e^{2 i \xi \cdot x}-\hat{V}(-2 \xi) e^{-2 i \xi \cdot x}\right],
\end{aligned}
$$

where, if necessary, the Fourier transform is regarded in the sense of tempered distributions, so that

$$
\begin{aligned}
\kappa & * \xi W(x, \xi)=\int_{\mathbb{R}^{n}} \kappa(x, \eta) W(x, \xi-\eta) d \eta \\
& =i \int_{\mathbb{R}^{n}}\left[V\left(x+\frac{y}{2}\right)-V\left(x-\frac{y}{2}\right)\right] \psi\left(x+\frac{y}{2}\right) \bar{\psi}\left(x-\frac{y}{2}\right) e^{-i \xi \cdot y} d y .
\end{aligned}
$$

Then $W=W[\psi]$ satisfies the Wigner equation:

$$
\partial_{t} W=-\xi \cdot \partial_{x} W-\kappa *_{\xi} W .
$$

Introducing the operator $A=-\xi \cdot \partial_{x}-\kappa * \xi$ the Wigner equation (7) can then be written in compact form as $\partial_{t} W=A W$. 
The following lemma then holds:

Lemma 1. For any $\phi \in \mathcal{S}\left(\mathbb{R}^{2 n}\right)$ we have

$$
\int \kappa *_{\xi} \phi d x d \xi=0
$$

Proof. Using (5) we can write

$$
\begin{aligned}
& \int \kappa *_{\xi} \phi d x d \xi= \\
& \quad=\frac{i}{\pi^{n}} \iiint\left[\hat{V}(2 \eta) e^{2 i \eta \cdot x}-\hat{V}(-2 \eta) e^{-2 i \eta \cdot x}\right] \phi(x, \xi-\eta) d \eta d \xi d x \\
& =\frac{i}{\pi^{n}} \int\left(\hat{V}(2 \eta) \int \mathcal{F}_{1} \phi(-2 \eta, \xi) d \xi-\hat{V}(-2 \eta) \int \mathcal{F}_{1} \phi(2 \eta, \xi) d \xi\right) d \eta
\end{aligned}
$$

where $\mathcal{F}_{1} \phi$ means the Fourier transform in the first variable of $\phi$, only. And obviously, this last integral is zero.

This immediately yields the next result, which characterizes the action of the operator $A$ on test functions, and will be useful in Section 4 .

Proposition 1. For all $\phi \in \mathcal{S}\left(\mathbb{R}^{2 n}\right)$

$$
\int A \phi(x, \xi) d x d \xi=0 \text {. }
$$

Furthermore, $A$ is skew-symmetric, that is,

$$
\langle\phi, A \psi\rangle=-\langle A \phi, \psi\rangle
$$

for all $\phi, \psi \in \mathcal{S}\left(\mathbb{R}^{2 n}\right)$.

Finally, the following quantities are conserved by the time evolution (7):

$$
\int_{\mathbb{R}^{2 n}} W d \xi d x, \int_{\mathbb{R}^{2 n}} W^{2} d \xi d x, \int_{\mathbb{R}^{2 n}}\left(\frac{|\xi|^{2}}{2}+V(x)\right) W d \xi d x .
$$

The first two, correspond to the conservation of $\|\psi\|_{2}$, whereas the third means that the average total energy stays constant with the flow.

\section{Exact time decay estimates}

In this section, we present two examples where explicit computations are possible: the free particle and the harmonic oscillator. In these, one can use the Wigner transform to establish elementary proofs of well known time decay inequalities for (3) since the Wigner equation (7) coincides precisely with the classical transport equation

$$
\partial_{t} W=-\xi \cdot \partial_{x} W+\partial_{x} V \cdot \partial_{\xi} W .
$$


Proposition 2. Let $\psi$ be the fundamental solution to the Schrödinger equation (3), with $V(x)=\alpha x^{2}$, with $\alpha=0, \pm 1$. Then, for $t>0$,

$$
\|\psi(\cdot, t)\|_{L_{x}^{\infty}} \leq \begin{cases}\frac{1}{(2 \pi t)^{n / 2}} & \text { if } \alpha=0, \\ \frac{1}{(2 \pi|\sin t|)^{n / 2}} & \text { if } \alpha=1, \\ \frac{1}{(2 \pi|\sinh t|)^{n / 2}} & \text { if } \alpha=-1 .\end{cases}
$$

Proof. The Wigner equation for $V(x)=\alpha x^{2}$ is

$$
W_{t}+\xi \cdot \partial_{x} W-\alpha x \cdot \partial_{\xi} W=0 .
$$

For the case $\alpha=0$, the free particle, we have the explicit representation formula for $W$ :

$$
W(x, \xi, t)=W(x-\xi t, \xi, 0) .
$$

This means that, for any test function $\phi$,

$$
\int \phi(x, \xi) W(x, \xi, t) d x d \xi=\int \phi(x+\xi t, \xi) W(x, \xi, 0) d x d \xi
$$

In the case $\alpha=+1$ define

$$
M(t)=\left[\begin{array}{cc}
\cos t & \sin t \\
-\sin t & \cos t
\end{array}\right]
$$

while, for $\alpha=-1$, define

$$
M(t)=\left[\begin{array}{cc}
\cosh t & \sinh t \\
-\sinh t & \cosh t
\end{array}\right]
$$

For these two cases, the solution satisfies

$$
\int \phi(x, \xi) W(x, \xi, t) d x d \xi=\int \phi\left(M(t)\left[\begin{array}{l}
x \\
\xi
\end{array}\right]\right) W(x, \xi, 0) d x d \xi .
$$

Now, notice that if the initial data is given by $f(x)=\delta\left(x-x_{0}\right)$ then we have

$$
W[f](x, \xi)=\delta\left(x-x_{0}\right) \otimes 1(\xi),
$$

where by $1(\xi)$ we mean the constant unit function in the $\xi$ variable. 
Therefore, if $\psi$ is the fundamental solution to the Schrödinger equation, for the case $\alpha=0$ observe that, for any $\phi \in C_{c}\left(\mathbb{R}^{n}\right)$, we get

$$
\begin{aligned}
\int \phi(x) \mid & \left.\psi(x, t)\right|^{2} d x= \\
= & \frac{1}{(2 \pi)^{n}} \int \phi(x)\left(\int W d \xi\right) d x=\frac{1}{(2 \pi)^{n}} \int \phi(x) W(\xi, x, t) d x d \xi \\
= & \frac{1}{(2 \pi)^{n}} \int \phi(x+\xi t) \delta(x) d x d \xi=\frac{1}{(2 \pi)^{n}} \int \phi(\xi t) d \xi \leq \frac{\|\phi\|_{L^{1}}}{(2 \pi t)^{n}}
\end{aligned}
$$

therefore yielding

$$
\|\psi(\cdot, t)\|_{L_{x}^{\infty}} \leq \frac{1}{(2 \pi t)^{n / 2}} .
$$

For the case $\alpha=+1$ we obtain, analogously,

$$
\begin{aligned}
& \frac{1}{(2 \pi)^{n}} \int \phi(x \cos t+\xi \sin t) \delta(x) d x d \xi= \\
&=\frac{1}{(2 \pi)^{n}} \int \phi(\xi \sin t) d \xi \leq \frac{\|\phi\|_{L^{1}}}{(2 \pi|\sin t|)^{n}}
\end{aligned}
$$

whereas for $\alpha=-1$ we have

$$
\begin{aligned}
& \frac{1}{(2 \pi)^{n}} \int \phi(x \cosh t+\xi \sinh t) \delta(x) d x d \xi= \\
&=\frac{1}{(2 \pi)^{n}} \int \phi(\xi \sinh t) d \xi \leq \frac{\|\phi\|_{L^{1}}}{(2 \pi|\sinh t|)^{n}}
\end{aligned}
$$

from which we conclude that $\|\psi\|_{L_{x}^{\infty}}$ is bounded by $\frac{1}{(2 \pi|\sin t|)^{n / 2}}$ and $\frac{1}{(2 \pi|\sinh t|)^{n / 2}}$, respectively for $\alpha=+1$ and $\alpha=-1$

\section{Estimates for the Wigner equation}

In this section we develop an abstract approach to the Wigner equation (17), and prove some estimates for phase space integral quantities associated to multiplication operators which localize the Wigner transform along the corresponding classical Hamiltonian evolution.

4.1. Abstract approach to the Wigner equation. Recall that if an operator $B$ is differentiable, then there exists a linear operator $B_{t}$, the derivative of $B$ with respect to the parameter $t$, such that

$$
\frac{d}{d t}(B \psi)=B_{t} \psi+B \psi_{t}
$$


Proposition 3. Let $B$ and $\tilde{A}$ be two operators, with $B W$ having enough decay at infinity, satisfying

$$
B_{t}+[B, \tilde{A}]=0
$$

Then

$$
\frac{d}{d t} \int B W=\int[B, A-\tilde{A}] W
$$

and

$$
\frac{d}{d t} \int|B W|^{2}=2 \operatorname{Re}(\langle B W,[B, A-\tilde{A}] W\rangle) .
$$

Proof. For the first identity, observe that

$\frac{d}{d t} \int B W=\int B_{t} W+B A W=\int B_{t} W+B A W-A B W=\int[B, A-\tilde{A}] W$,

while, for the second, we have

$$
\begin{aligned}
\frac{d}{d t} \int|B W|^{2}= & \langle B W, B A W\rangle+\langle B A W, B W\rangle+\left\langle B W, B_{t} W\right\rangle+\left\langle B_{t} W, B W\right\rangle \\
= & \langle B W,[B, A] W\rangle+\langle B W, A B W\rangle+\langle[B, A] W, B W\rangle \\
& +\langle A B W, B W\rangle-\langle B W,[B, \tilde{A}] W\rangle-\langle[B, \tilde{A}] W, B W\rangle \\
= & \langle B W,[B, A-\tilde{A}] W\rangle+\langle[B, A-\tilde{A}] W, B W\rangle .
\end{aligned}
$$

As Corollaries of these two indentities we have

Corollary 1. Under the same conditions as above

$$
\left|\frac{d}{d t}\|B W\|_{L^{2}}\right| \leq\|[B, A-\tilde{A}] W\|_{L^{2}} .
$$

Corollary 2. Let $B$ be a constant operator that commutes with $A$. Then

$$
\frac{d}{d t} \int B W=0 \quad \frac{d}{d t} \int|B W|^{2}=0 .
$$

In particular if $B=A^{k}$ we have $\frac{d}{d t} \int A^{k} W=0$, and $\frac{d}{d t} \int\left|A^{k} W\right|^{2}=0$.

Proof. Just make $\tilde{A}=0$.

In the case $k=0$, the last corollary yields

$$
\frac{d}{d t} \int W=\frac{d}{d t} \int W^{2}=0
$$


4.2. Commutator equations. Let us consider the classical approximation $\tilde{A}$ to $A$, that is

$$
\tilde{A}=-\xi \cdot \partial_{x}+\partial_{x} V \cdot \partial_{\xi}
$$

An elementary computation shows that $\tilde{A}$ satisfies the properties that are stated for the Wigner operator $A$ in Proposition 11. Let $B$ be a differential operator of order $m$. Since $\tilde{A}$ is a first-order differential operator, then $[B, \tilde{A}]$ has order at most $m$.

Suppose $B$ satisfies $B_{t}+[B, \tilde{A}]=0$. In the particular case of multiplication operators $B \psi=\phi \psi$ this condition is satisfied if $\phi$ is a solution to the classical transport equation

$$
\phi_{t}=\tilde{A} \phi=-\xi \cdot \partial_{x} \phi+\partial_{x} V \cdot \partial_{\xi} \phi .
$$

Consider the transport equation (17). The explicit representation formula for the solutions is

$$
\phi(x, \xi, t)=\phi\left(\Theta_{t}^{-1}(x, \xi), 0\right),
$$

where $\Theta_{t}$ is the flow generated by the Hamiltonian equations

$$
\dot{x}=\xi \quad \dot{\xi}=-\partial_{x} V .
$$

In particular, this implies that all $L^{p}$ norms of $\phi$ are conserved. Furthermore, the smoothness of the solutions can be estimated from the initial data and the flow map $\Theta$.

4.3. Estimates for multiplication operators. Now we address the problem of estimating the time-evolution of the norms of multiplication operators acting on the Wigner transform. From now on we consider $\phi$ to be a solution of the classical transport equation $\phi_{t}=\tilde{A} \phi$. If the Wigner transform turns out to be also a solution to $W_{t}=\tilde{A} W$, i.e. if the Wigner equation (7) reduces to (10) then

$$
\frac{d}{d t} \int|W-\phi|^{2}=-2 \frac{d}{d t} \int \phi W=0,
$$

and

$$
\frac{d}{d t} \int \phi^{2} W^{2}=0
$$

Unfortunately, this only happens for the (very special) cases of the harmonic oscillator or the free particle considered in the previous section. In general these identities are false, and, therefore, one would like to obtain quantitative estimates both for $\frac{d}{d t} \int \phi W$, which would control the evolution of the $L^{2}$ norm of the difference, and for $\frac{d}{d t} \int \phi^{2} W^{2}$, which is helpful in 
tracking the localization of the Wigner transform, when it evolves according to (7) but as seen from a "window" with Hamiltonian evolution, given by $\phi$.

Theorem 1. Let $B$ be a multiplication operator $B W=\phi W$, with $\phi \in$ $\mathcal{S}\left(\mathbb{R}^{2 n}\right)$, such that $B_{t}+[B, \tilde{A}]=0$, i.e. satisfying (17). Then:

$$
\frac{d}{d t} \int \phi W=\int-\phi k *_{\xi} W+W\left(\partial_{x} V \cdot \partial_{\xi} \phi\right) .
$$

Furthermore, the right-hand side of (19) is bounded by

$$
\left|\int \phi k *_{\xi} W-W\left(\partial_{x} V \cdot \partial_{\xi} \phi\right)\right| \leq C_{b}\|W\|_{L^{2}}\left\|\partial_{\xi \xi}^{2} \phi\right\|_{L^{2}},
$$

where the constant $C_{b}$ depends on the perturbation $b(x)$ of the basic quadratic potential.

Proof. From Proposition 3 and Lemma 1 one easily obtains (19).

Then observe that

$$
\begin{aligned}
& \iint \phi k *_{\xi} W-W\left(\partial_{x} V \cdot \partial_{\xi} \phi\right) d \xi d x \\
& =\frac{i}{(2 \pi)^{n}} \iiint \int\left[V\left(x+\frac{y}{2}\right)-V\left(x-\frac{y}{2}\right)\right. \\
& \left.\quad-y \cdot \partial_{x} V(x)\right] e^{-i(\xi-\eta) \cdot y} W(x, \eta) \phi(x, \xi) d \xi d y d \eta d x \\
& =\frac{i}{(2 \pi)^{n}} \iiint \iint_{0}^{1} \int_{0}^{1} \frac{\theta}{4} \sum_{j, k}\left[\partial_{x_{j} x_{k}}^{2} V\left(x+\frac{\theta \delta y}{2}\right)\right. \\
& \left.\quad-\partial_{x_{j} x_{k}}^{2} V\left(x-\frac{\theta \delta y}{2}\right)\right] y_{j} y_{k} e^{-i(\xi-\eta) \cdot y} W(x, \eta) \phi(x, \xi) d \delta d \theta d \xi d y d \eta d x \\
& =-\frac{i}{(2 \pi)^{n}} \iiint \iint_{0}^{1} \int_{0}^{1} \frac{\theta}{4} \sum_{j, k}\left[\partial_{x_{j} x_{k}}^{2} V\left(x+\frac{\theta \delta y}{2}\right)\right. \\
& \left.\quad-\partial_{x_{j} x_{k}}^{2} V\left(x-\frac{\theta \delta y}{2}\right)\right] e^{-i(\xi-\eta) \cdot y} W(x, \eta) \partial_{\xi_{j}}^{2} \xi_{k} \phi(x, \xi) d \delta d \theta d \xi d y d \eta d x .
\end{aligned}
$$

Denote by

$g_{j, k}(x, z)=\partial_{x_{j} x_{k}}^{2} V(x+z)-\partial_{x_{j} x_{k}}^{2} V(x-z)=\partial_{x_{j} x_{k}}^{2} b(x+z)-\partial_{x_{j} x_{k}}^{2} b(x-z)$. 
Then, the right-hand side term of the previous identity can be written as

$$
\begin{gathered}
-\frac{i}{(2 \pi)^{n}} \iiint \iint_{0}^{1} \int_{0}^{1} \frac{\theta}{4} \sum_{j, k} g_{j, k}\left(x, \frac{\theta \delta y}{2}\right) e^{-i(\xi-\eta) \cdot y} \\
W(x, \eta) \partial_{\xi_{j} \xi_{k}}^{2} \phi(x, \xi) d \delta d \theta d \xi d y d \eta d x \\
=-\frac{i}{(2 \pi)^{n}} \iiint \int_{0}^{1} \int_{0}^{1} \frac{\theta}{4} \sum_{j, k}\left(\frac{\theta \delta}{2}\right)^{-n} \mathcal{F}_{z}\left(g_{j, k}\right)\left(x, \frac{2(\xi-\eta)}{\theta \delta}\right) \\
W(x, \eta) \partial_{\xi_{j} \xi_{k}}^{2} \phi(x, \xi) d \delta d \theta d \xi d \eta d x \\
=\iint \sum_{j, k} \tilde{W}_{j, k}(x, \xi) \partial_{\xi_{j}}^{2} \xi_{k} \phi(x, \xi) d \xi d x,
\end{gathered}
$$

where

$$
\begin{aligned}
& \tilde{W}_{j, k}(x, \xi)= \\
& -\frac{i}{(2 \pi)^{n}} \iint_{0}^{1} \int_{0}^{1}\left(\frac{\theta \delta}{2}\right)^{-n} \mathcal{F}_{z}\left(g_{j, k}\right)\left(x, \frac{2(\xi-\eta)}{\theta \delta}\right) W(x, \eta) d \delta d \theta d \eta,
\end{aligned}
$$

and $\mathcal{F}_{z}$ denotes the Fourier transform in the $z$ variable,

$$
\mathcal{F}_{z}\left(g_{j, k}\right)(x, \zeta)=\zeta_{j} \zeta_{k}\left[\hat{b}(\zeta) e^{i x \cdot \zeta}-\hat{b}(-\zeta) e^{-i x \cdot \zeta}\right] .
$$

Since $\left(\frac{\theta \delta}{2}\right)^{-n} \mathcal{F}_{z}\left(g_{j, k}\right)\left(x, \frac{2 \xi}{\theta \delta}\right)$ is bounded uniformly in $L_{\xi}^{1}$, for all $x$, we obtain $\|\tilde{W}\|_{L^{2}} \leq C\|W\|_{L^{2}}$, and then the estimate follows.

Theorem 2. Under the same hypotheses as in Theorem 1,

$$
\begin{aligned}
\|[B, A-\tilde{A}] W\|_{L^{2}} \leq & \left\|\left(\partial_{x} V \cdot \partial_{\xi} \phi+\kappa *_{\xi} \phi\right) W\right\|_{L^{2}} \\
& +\left\|\left(\kappa *_{\xi} W\right) \phi+\left(\kappa *_{\xi} \phi\right) W-\kappa *_{\xi}(\phi W)\right\|_{L^{2}} .
\end{aligned}
$$

Furthermore,

$$
\begin{array}{r}
\left\|\left(\kappa *_{\xi} W\right) \phi+\left(\kappa *_{\xi} \phi\right) W-\kappa *_{\xi}(\phi W)\right\|_{L^{2}} \\
\leq C\left(\left\|\partial_{x} b\left(x+\frac{y}{2}\right)+\partial_{x} b\left(x-\frac{y}{2}\right)-2 \partial_{x} b(x)\right\|_{L_{x, y}^{\infty}}\left\|\mathcal{F}_{\xi}\left(\partial_{\xi} \phi\right)\right\|_{L_{z}^{1}\left(L_{x}^{2}\right)}\right. \\
\left.+\left\|\mathcal{F}_{\xi}\left(\partial_{\xi}^{2} \phi\right)\right\|_{L_{z}^{1}\left(L_{x, y}^{\infty}\right)}\right)\|W\|_{L^{2}}
\end{array}
$$

and 


$$
\left\|\left(\partial_{x} V \cdot \partial_{\xi} \phi+\kappa *_{\xi} \phi\right) W\right\| \leq C\left\|\partial_{\xi \xi}^{2} \phi\right\|_{L^{2}}\|W\|_{L^{2}},
$$

where $\mathcal{F}_{\xi}$ is the partial Fourier transform in the $\xi$ variable only, and $z$ is the dual variable to $\xi$.

Proof. First note that $A-\tilde{A}=-\kappa *_{\xi}-\partial_{x} V \cdot \partial_{\xi}$. Then

$$
\begin{aligned}
{[B, A-\tilde{A}] W=\phi(A-\tilde{A}) W } & -(A-\tilde{A})(\phi W)= \\
& =\left(\partial_{x} V \cdot \partial_{\xi} \phi\right) W-\left(\kappa *_{\xi} W\right) \phi+\kappa *_{\xi}(\phi W),
\end{aligned}
$$

and so

$$
\begin{aligned}
\|[B, A-\tilde{A}] W\|_{L^{2}} \leq & \left\|\left(\partial_{x} V \cdot \partial_{\xi} \phi\right) W+\left(\kappa *_{\xi} \phi\right) W\right\|_{L^{2}} \\
& +\left\|\left(\kappa *_{\xi} W\right) \phi+\left(\kappa *_{\xi} \phi\right) W-\kappa *_{\xi}(\phi W)\right\|_{L^{2}} .
\end{aligned}
$$

Note that, by using a partial Fourier transform in the variable $\xi$, we have

$$
\begin{aligned}
& \left\|\left(\kappa *_{\xi} W\right) \phi+\left(\kappa *_{\xi} \phi\right) W-\kappa *_{\xi}(\phi W)\right\|_{L_{x, \xi}^{2}} \\
& \quad=\frac{1}{(2 \pi)^{\frac{3 n}{2}}}\left\|(\hat{\kappa} \hat{W}) *_{y} \hat{\phi}+(\hat{\kappa} \hat{\phi}) *_{y} \hat{W}-\hat{\kappa}\left(\hat{\phi} *_{y} \hat{W}\right)\right\|_{L_{x, y}^{2}}
\end{aligned}
$$

where the variable $y$ is the dual variable corresponding to $\xi$. The convolutions on the right-hand side of the previous identity can be written as

$$
\begin{aligned}
& \int_{\mathbb{R}^{n}}[\hat{\kappa}(x, y-z) \hat{\phi}(x, z) \hat{W}(x, y-z)+\hat{\kappa}(x, z) \hat{\phi}(x, z) \hat{W}(x, y-z) \\
& \quad-\hat{\kappa}(x, y) \hat{\phi}(x, z) \hat{W}(x, y-z)] d z \\
& =\int_{\mathbb{R}^{n}}[\hat{\kappa}(x, y-z)-\hat{\kappa}(x, y)+\hat{\kappa}(x, z)] \hat{\phi}(x, z) \hat{W}(x, y-z) d z .
\end{aligned}
$$

Recall that $\hat{\kappa}(x, y)=i\left(V\left(x-\frac{y}{2}\right)-V\left(x+\frac{y}{2}\right)\right)$, therefore

$$
\hat{\kappa}(x, y-z)-\hat{\kappa}(x, y)=i\left(\partial_{x} V\left(x+\frac{y}{2}\right) \cdot \frac{z}{2}+\partial_{x} V\left(x-\frac{y}{2}\right) \cdot \frac{z}{2}\right)+O\left(|z|^{2}\right),
$$

and

$$
\hat{\kappa}(x, z)=-i \partial_{x} V(x) \cdot z+O\left(|z|^{2}\right) .
$$


Note that the $O\left(|z|^{2}\right)$ error term is uniform in $x, y$. Therefore,

$$
\begin{aligned}
\|\left(\kappa *_{\xi} W\right) \phi+ & \left(\kappa *_{\xi} \phi\right) W-\kappa *_{\xi}(\phi W) \|_{L_{x, \xi}^{2}} \\
\lesssim \| \int_{\mathbb{R}^{n}} \frac{i\left[\partial_{x} V\left(x+\frac{y}{2}\right)+\partial_{x} V\left(x-\frac{y}{2}\right)-2 \partial_{x} V(x)\right]}{2} & \cdot z \hat{\phi}(x, z) \hat{W}(x, y-z) d z \|_{L_{x, y}^{2}} \\
& +\left\|\int_{\mathbb{R}^{n}} O\left(|z|^{2}\right) \hat{\phi}(x, z) \hat{W}(x, y-z) d z\right\|_{L_{x, y}^{2}} .
\end{aligned}
$$

First, observe that

$\partial_{x} V\left(x+\frac{y}{2}\right)+\partial_{x} V\left(x-\frac{y}{2}\right)-2 \partial_{x} V(x)=\partial_{x} b\left(x+\frac{y}{2}\right)+\partial_{x} b\left(x-\frac{y}{2}\right)-2 \partial_{x} b(x)$, so that using the integral version of the Minkowski inequality and Plancherel

$$
\begin{gathered}
\| \int_{\mathbb{R}^{n}} \frac{i\left[\partial_{x} V\left(x+\frac{y}{2}\right)+\partial_{x} V\left(x-\frac{y}{2}\right)-2 \partial_{x} V(x)\right]}{2} \\
\cdot z \hat{\phi}(x, z) \hat{W}(x, y-z) d z \|_{L_{x, y}^{2}} \\
\leq\left\|\int_{\mathbb{R}^{n}}\right\| \partial_{x} b\left(x+\frac{y}{2}\right)+\partial_{x} b\left(x-\frac{y}{2}\right)-2 \partial_{x} b(x) \|_{L_{y}^{\infty}} \\
\cdot\|\hat{W}(x, y)\|_{L_{y}^{2}} \widehat{\partial_{\xi} \phi}(x, z) \mid d z \|_{L_{x}^{2}} \\
\lesssim\left\|\partial_{x} b\left(x+\frac{y}{2}\right)+\partial_{x} b\left(x-\frac{y}{2}\right)-2 \partial_{x} b(x)\right\|_{L_{x, y}^{\infty}} \\
\cdot\|W\|_{L_{x, \xi}^{2}} \int_{\mathbb{R}^{n}}\left\|\widehat{\partial_{\xi} \phi}(x, z)\right\|_{L_{x}^{2}} d z .
\end{gathered}
$$

As for the second term in (20), we denote by $\chi(x, y, z)=O\left(|z|^{2}\right) \hat{\phi}(x, z)$ and again use Minkowski and Plancherel. Then

$$
\begin{aligned}
\left\|\int_{\mathbb{R}^{n}} O\left(|z|^{2}\right) \hat{\phi}(x, z) \hat{W}(x, y-z) d z\right\|_{L_{x, y}^{2}} \\
\leq \int_{\mathbb{R}^{n}}\|\chi(x, y, z) \hat{W}(x, y-z)\|_{L_{x, y}^{2}} d z \\
\leq \int_{\mathbb{R}^{n}}\|\chi(x, y, z)\|_{L_{x, y}^{\infty}}\|\hat{W}(x, y)\|_{L_{x, y}^{2}} d z \\
\leq C\|\chi(x, y, z)\|_{L_{z}^{1}\left(L_{x, y}^{\infty}\right)}\|W(x, \xi)\|_{L_{x, \xi}^{2}}
\end{aligned}
$$


Thus,

$$
\left\|\int_{\mathbb{R}^{n}} O\left(|z|^{2}\right) \hat{\phi}(x, z) \hat{W}(x, y-z) d z\right\|_{L_{x, y}^{2}} \leq C\left\|\widehat{\partial_{\xi}^{2} \phi}\right\|_{L_{z}^{1}\left(L_{x, y}^{\infty}\right)}\|W\|_{L_{x, \xi}^{2}} .
$$

To prove the last estimate of the theorem, observe that

$$
\begin{aligned}
\kappa *_{\xi} \phi=\frac{i}{(2 \pi)^{n}} \int_{\mathbb{R}^{2 n}}\left[V\left(x+\frac{y}{2}\right)-V\left(x-\frac{y}{2}\right)\right] e^{-i \eta \cdot y} \phi(x, \xi-\eta) d \eta d y \\
=\frac{i}{(2 \pi)^{n}} \int_{\mathbb{R}^{2 n}} \partial_{x} V(x) \cdot y e^{-i \eta \cdot y} \phi(x, \xi-\eta) d \eta d y \\
\quad+\frac{i}{(2 \pi)^{n}} \int_{\mathbb{R}^{2 n}} \chi(x, y) e^{-i \eta \cdot y} \phi(x, \xi-\eta) d \eta d y,
\end{aligned}
$$

where $|\chi(x, y)| \leq C|y|^{2}$. Note that

$$
\frac{i}{(2 \pi)^{n}} \int_{\mathbb{R}^{2 n}} \partial_{x} V(x) \cdot y e^{-i \eta \cdot y} \phi(x, \xi-\eta) d \eta d y=-\partial_{x} V(x) \cdot \partial_{\xi} \phi(x, \xi) .
$$

Therefore it suffices to estimate the last term. Again using Plancherel, we have

$$
\begin{aligned}
\| \int_{\mathbb{R}^{2 n}} \chi & (x, y) e^{-i \eta \cdot y} \phi(x, \xi-\eta) d \eta d y \|_{L_{x, \xi}^{2}} \\
& =\left\|\int_{\mathbb{R}^{n}} \frac{\chi(x, y)}{|y|^{2}} e^{-i \xi \cdot y}|y|^{2} \hat{\phi}(x,-y) d y\right\|_{L_{x, \xi}^{2}} \\
& =(2 \pi)^{(n / 2)}\left\|\frac{\chi(x, y)}{|y|^{2}}|y|^{2} \hat{\phi}(x,-y)\right\|_{L_{x, y}^{2}} \leq C\left\|\partial_{\xi \xi}^{2} \phi\right\|_{L_{x, \xi}^{2}} .
\end{aligned}
$$

\section{References}

[1] T. Colin, "Smoothing effects for dispersive equations via a generalized Wigner transform", SIAM Journal on Mathematical Analysis 25, 6, (1994), pp. 16221641.

[2] G. Folland, Harmonic Analysis in Phase Space, Princeton University Press, Princeton, N.J., 1989.

[3] I. Gasser, P.A. Markowich and B. Perthame, "Dispersion and moment lemmas revisited", Journal of Differential Equations 156, (1999), pp. 254-281.

[4] P. Gérard, P.A. Markowich, N.J. Mauser and F. Poupaud, "Homogenization limits and Wigner transforms", Communications on Pure and Applied Mathematics 50, 4, (1997), pp. 323-379.

[5] P.L. Lions and T. Paul, "Sur les mesures de Wigner", Revista Matemática Iberoamericana 9, 3, (1993), pp. 553-618. 